\title{
Cooling and Collisions of Large Gas Phase Molecules
}

\section{Citation}

Patterson, David, Edem Tsikata, and John M. Doyle. 2010. Cooling and collisions of large gas phase molecules. Physical Chemistry Chemical Physics 12(33): 9736-9741.

\section{Published Version}

doi:10.1039/c002764b

\section{Permanent link}

http://nrs.harvard.edu/urn-3:HUL.InstRepos:8866867

\section{Terms of Use}

This article was downloaded from Harvard University's DASH repository, and is made available under the terms and conditions applicable to Other Posted Material, as set forth at http:// nrs.harvard.edu/urn-3:HUL.InstRepos:dash.current.terms-of-use\#LAA

\section{Share Your Story}

The Harvard community has made this article openly available.

Please share how this access benefits you. Submit a story.

\section{Accessibility}




\title{
Cooling and collisions of large gas phase molecules
}

\author{
David Patterson," Edem Tsikata and John M. Doyle
}

\author{
Received 9th February 2010, Accepted 21st April 2010 \\ DOI: $10.1039 / \mathbf{c 0 0 2 7 6 4 b}$
}

Cold and dense samples of naphthalene $\left(\mathrm{C}_{10} \mathrm{H}_{8}\right)$ are produced using buffer gas cooling in combination with rapid, high flow molecule injection. The observed naphthalene density is $n \approx 10^{11} \mathrm{~cm}^{-3}$ over a volume of a few $\mathrm{cm}^{3}$ at a temperature of $6 \mathrm{~K}$. We observe naphthalene-naphthalene collisions through two-body loss of naphthalene with a loss cross section of $\sigma_{\mathscr{N}-\mathscr{N}}=1.4 \times 10^{-14} \mathrm{~cm}^{2}$. Analysis is presented that indicates that this combination of techniques will be applicable to many comparably sized molecules. This technique can also be combined with cryogenic beam methods ${ }^{1}$ to produce cold, high flux, continuous molecular beams.

\section{Introduction}

Driven by a variety of new science, including cold chemistry and dipolar quantum gases, several methods are now being pursued to produce cold and ultracold samples of molecules. The cold molecules in these studies are generally diatomic but also include few-atom molecules such as $\mathrm{ND}_{3}{ }^{2,3}$ Extending this work to the cooling of larger molecules is of high interest, as reviewed by Meijer et l. $^{4}$ and references therein. For example, chemical reaction rates at low temperatures are of great current interest, and extending these studies to important large molecules is essential. Similarly, ultraprecise spectroscopy applications could make use of new continuous cryogenic molecular beams. Finally, there is great interest in field mediated chemistry, and a general source of high density, highly polarizable ground state molecules is an excellent testbed for observing field mediated chemical reactions.

In previous work with buffer gas cooling, cold, guided beams of molecules as large as trifluoromethane $\left(\mathrm{CF}_{3} \mathrm{H}\right)$ have been produced. ${ }^{1,5}$ In related work, a beam of slow $\left(11 \mathrm{~m} \mathrm{~s}^{-1}\right)$, but rotationally and vibrationally warm $(300 \mathrm{~K})$, Perfluoro $\mathrm{C}_{60}$ (mass $>6000 \mathrm{amu}$ ) was produced by filtering slow molecules from a warm sample. ${ }^{6}$ Cold samples of large molecular ions are routinely produced and trapped. However, the only demonstrated technique for producing samples of neutral cold molecules with atom number higher than five is the seeded supersonic jet. Seeded supersonic beams have a rich history and have found great utility in spectroscopic studies, ${ }^{7}$ as sources for molecular trapping experiments, ${ }^{4}$ and in cold chemistry experiments using the CRESU technique. ${ }^{8}$ They are limited, however, because although they produce translationally and rotationally cold molecules, these molecules are moving at very high velocity $\left(300 \mathrm{~m} \mathrm{~s}^{-1}\right.$ or higher). Furthermore, the beam evolves spatially with a rapidly decreasing density as the molecules move away from the beam orifice.

New cold beam methods (some mentioned just above) are part of a renaissance in molecular beams. Part of this renaissance

Physics Department, Harvard University, 17 Oxford Street, Cambridge, MA 02138,USA.E-mail: davepatterson99@gmail.com; Tel: +16174952501 is the use of electric and magnetic fields to manipulate and decelerate polar and magnetic molecules. In many cases, these methods are applied to molecules in metastable states, such as low-field seeking electric dipole states. Extending these slowing and trapping methods to larger molecules is inherently problematic because, unlike diatomics and few-atom molecules, polyatomic molecules effectively have only high field seeking states due to their small rotational splittings. This nearly eliminates low-field seeking electric guides and decelerators from applicability. To overcome these constraints, ingenious techniques have been fielded to align ${ }^{9,10}$ separate, ${ }^{11}$ and decelerate $^{4}$ larger molecules. Great progress has been made manipulating these molecules, but producing samples of very large, cold molecules at rest in the laboratory frame has remained elusive.

Here we report on the creation of gas-phase naphthalene at $6 \mathrm{~K}$, created through a novel rapid helium gas cooling method. Prior to this work, it was an open question whether larger molecules in a cryogenic helium gas would rapidly accumulate a layer of bound helium atoms or instead remain "naked". In the latter case the naphthalene would cool to the helium temperature but remaining free of adsorbed helium atoms. It is easy to see why clustering could be expected-the high binding energy of a helium atom to the molecule, in combination with the rich vibrational mode structure of larger molecules and the low temperature of the helium gas, could lead to the creation of long lived excited molecule-helium dimers, which would initiate clustering. The creation of $6 \mathrm{~K}$ naphthalene described in this work demonstrates that this is not the case, and thus greatly increases the potential scope of buffer gas cooling as a production method for cold, gas phase molecules. To our knowledge, this work represents the first observation of cold $(<10 \mathrm{~K})$, larger ( $>5$ atoms) molecules that are moving slowly in the laboratory rest frame. As we will describe in detail, in addition to cooling naphthalene and placing limits on the He-naphthalene clustering rate, we were also able to observe the loss of naked naphthalene due to cluster initiation from two body naphthalene-naphthalene collisions. Our studies offer evidence that rapid clustering in a cryogenic gas depends critically on the vibrational properties of the cluster constituents. A simple model suggests that our cooling methodology will be 
applicable to a wide variety of molecules of size comparable to (or smaller than) naphthalene.

\subsection{Apparatus}

A cold mixture of naphthalene $\left(\mathrm{C}_{10} \mathrm{H}_{8}\right.$, denoted $\mathscr{N}$ in this paper) and helium is produced by injecting a hot $(300 \mathrm{~K})$ mixture into a cold cell tube via a short $(1 \mathrm{~cm})$, thermally isolating transition tube. Our apparatus, shown in Fig. 1, is a qualitative change from the cooling system described in ref. 1. In that work, cold beams of potassium and ammonia were produced by cooling entrained mixtures with neon buffer gas and flowing the mixture through an aperture into vacuum. In this work, the beam aperture was replaced by a pumping line. The lower oven temperature and design improvements allow us to use a significantly shorter transition tube in comparison with earlier work, reducing input losses and allowing us to run with substantially lower buffer gas flows, leading to lower in-cell helium densities. These crucial changes allowed for the success of these experiments.

The mixture begins to cool as soon as it enters the cold cell tube. Any molecule which diffuses to a cold wall sticks and is lost from the gas. A species $A$ entrained in the buffer gas will cool with little loss as long as the elastic scattering cross section $\sigma_{\mathrm{A}-\mathrm{He}}>\sigma_{\mathrm{He}-\mathrm{He}}$. Although the low temperature cross section $\sigma_{\mathscr{N}-H e}$ is not known, the success of this experiment indicates that this criteria appears to be met for naphthalene, in agreement with the basic expectation that a naphthalene molecule is physically larger than a helium atom. As the mixture enters the cell and thermalizes, the phase space density of naphthalene increases by more than 5 orders of magnitude. This is due to the combination of rotational cooling, translational cooling, and physical compression that takes place within the cold cell. Ref. 1 and 12 contain detailed descriptions of the dynamics of this system.

Cold naphthalene is detected using laser induced fluorescence, excited by a pulsed laser $(10 \mathrm{~Hz}, 2 \mu \mathrm{J}, 308.0 \mathrm{~nm})$ that is fired along the tube axis. The laser can be tuned across either the strong $8_{0}^{1}$ transition or the weaker $8_{0}^{0}$ origin transition of neutral naphthalene. Fluorescence is collected through quartz windows by PMTs on each side of the cell. One PMT collects light from the upstream half of the cell, while the other only collects from the downstream half; comparison of these two spectra allows for direct measurements of loss and cooling as the gas mixture passes through the cell.

\section{Results}

A typical LIF spectrum of cold naphthalene $\left(n=2 \times 10^{11} \mathrm{~cm}^{-3}\right.$, $T_{\text {cell }}=6.2 \mathrm{~K}$ ) is shown in Fig. 2, along with a theoretical curve fit to $6.2 \mathrm{~K} \pm 1.5 \mathrm{~K}$. The absolute density of the naphthalene is calibrated as follows: under certain, set conditions, it is found that naphthalene loss from one end of the cell tube to the other is small. The cell tube is longer than the transition tube, and so the assumption is made that loss in the transition tube is also small. This allows us to estimate the cold density from the known input density, and the fluorescence is calibrated using

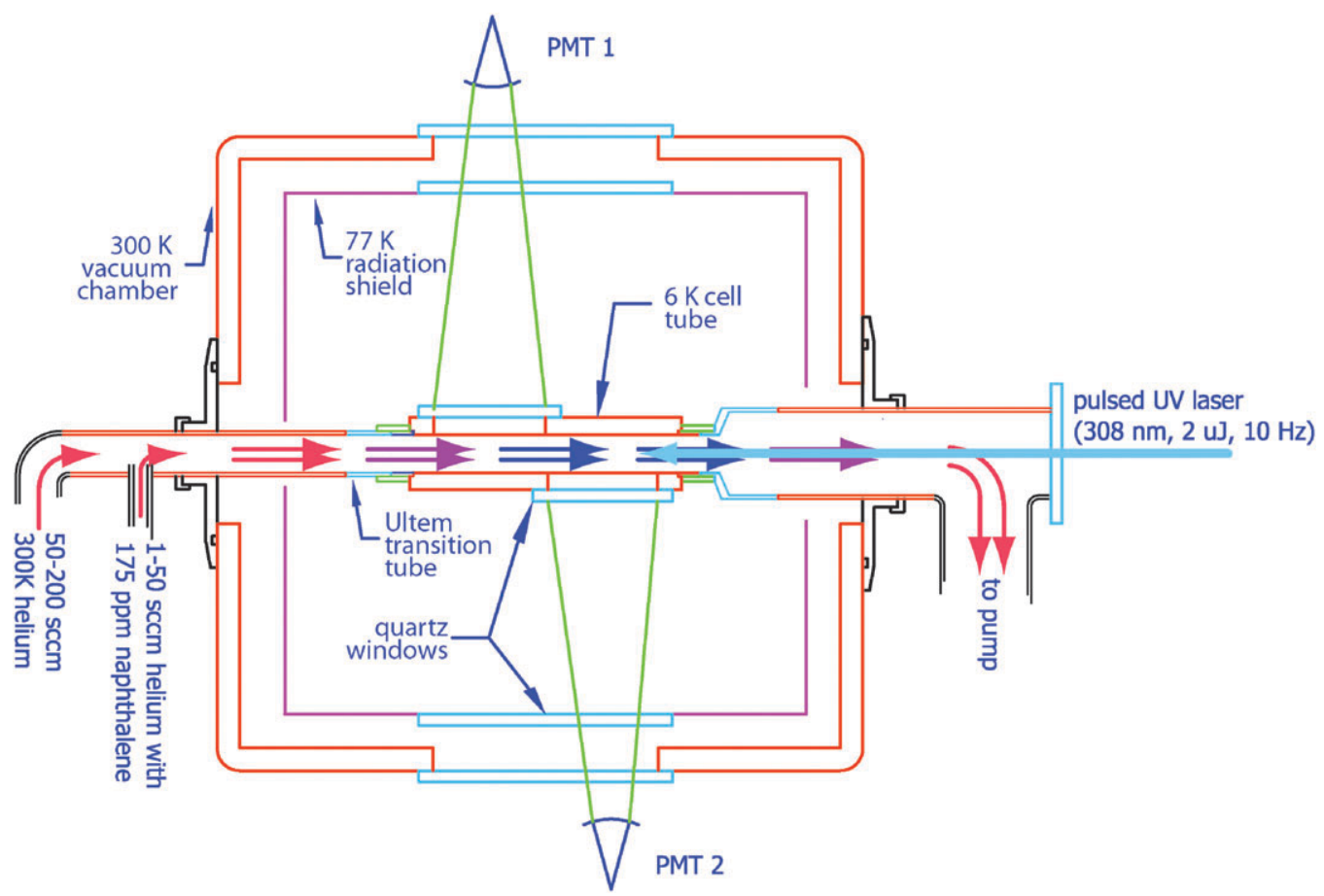

Fig. 1 A mixture of helium buffer gas and naphthalene flows down a tube from $300 \mathrm{~K}$, where the naphthalene has significant vapor pressure, to a cryogenic cell anchored to a liquid helium bath. Considerable care is taken to keep the transition region as short as possible while maintaining an adequate thermal disconnect. This is achieved by an Ultem ${ }^{\circledR}$ tube, length $10 \mathrm{~mm}$, wall thickness $0.5 \mathrm{~mm}$, between the $300 \mathrm{~K}$ input tube and the cold cell tube. The cell tube is thermally anchored to the helium bath of a small cryostat; it is then connnected via a second Ultem ${ }^{\circledR}$ tube to a $2 \mathrm{~cm}$ diameter pumping line. The total heat load on the helium bath with no buffer gas flowing is $\approx 500 \mathrm{~mW}$, probably dominated by blackbody radiation from the $300 \mathrm{~K}$ pumpout line; with a typical flow of $100 \mathrm{sccm}$ of helium, this heat load increases to $\approx 900 \mathrm{~mW}$. The helium pressure is 2 Torr at the tube input and $150 \mathrm{mTorr}$ at the tube output. 


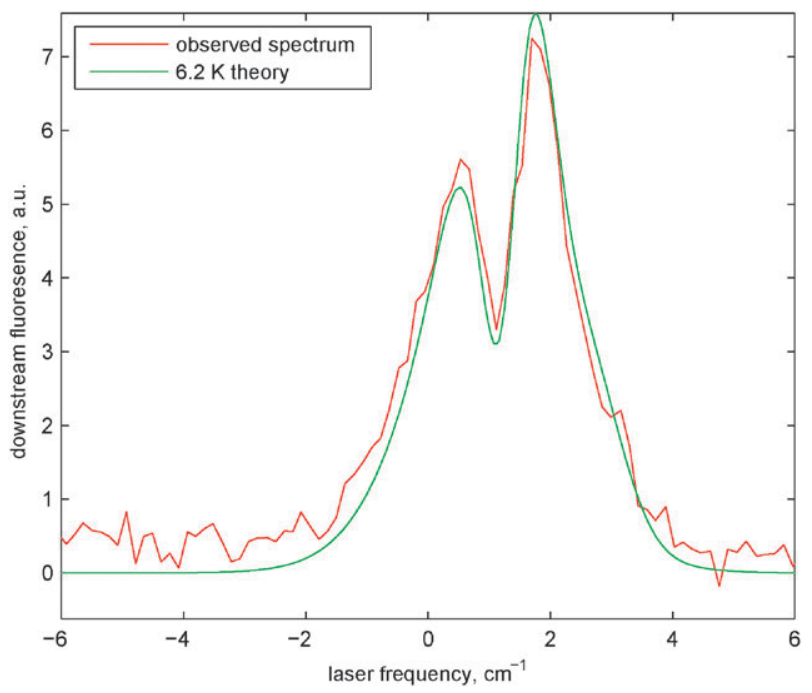

Fig. 2 Laser induced fluorescence from $8_{0}^{1} \operatorname{transition}(\lambda=308.1 \mathrm{~nm})$ of cold naphthalene (density $n \approx 2 \times 10^{11} \mathrm{~cm}^{-3}, T=6.2 \mathrm{~K}$ ). Fluorescence was collected by the photomultiplier observing only the downstream (colder) half of the flow tube. The naphthalene observed here has cooled from $300 \mathrm{~K}$ to $6 \mathrm{~K}$ with minimal (<factor 5 ) loss to the cell walls. A curve fit to a theoretical spectrum with $T=6.2 \mathrm{~K}$ is shown; the uncertainty in this fit is $\pm 1.5 \mathrm{~K}$. The theory is an exact calculation of all rotational lines with $J<100^{13} .^{12}$

this value. This calibration is checked to be consistent with a second calibration made at room temperature. In this case, the naphthalene density is known because naphthalene will not accumulate on the walls at $300 \mathrm{~K}$. The helium density is calculated from the pressure, which is bounded by the measured pressures at the input and output of the flow tube. The rotational temperature of the naphthalene is measured by comparing the measured spectrum with an exact calculation of all rotational lines with $J<100 .^{13}$

We are thus able to determine the density of naphthalene and helium separately, through calibrated LIF and direct pressure measurement, respectively. We find that under typical conditions, the cell contains helium at a density of $n_{\mathrm{He}}=$ $4 \times 10^{17} \mathrm{~cm}^{-3}$ and naphthalene at a density of $n_{\mathcal{N}}=5 \times$ $10^{11} \mathrm{~cm}^{-3}$, at a temperature of $T=6.2 \mathrm{~K}$. Uncertainties in our understanding of naphthalene loss mechanisms and of the helium pressure within the cold cell tube limit the certainty of the absolute naphthalene density measurement to an order of magnitude. The cold naphthalene is entrained in the moving helium flow, with flow velocity is $v_{\text {flow }} \approx 1.5 \mathrm{~m} \mathrm{~s}^{-1}$, compared to a microscopic thermal velocity $c=\sqrt{2 k_{b} T / m}=30 \mathrm{~m} \mathrm{~s}^{-1}$ and typical seeded supersonic beam velocities of $>300 \mathrm{~m} \mathrm{~s}^{-1}$. Fig. 2 therefore represents naphthalene molecules which are effectively at rest in the lab frame.

\subsection{Loss mechanisms and experimental results}

We are able to study the effects of naphthalene collisions by measuring the loss of naphthalene as it is transported through the flow tube at low velocity. Under typical running conditions, a substantial order of unity fraction of the naphthalene disappears as it is swept from the first half of the tube (upstream signal) into the second half (downstream signal). Fig. 3 shows these signals as a function of naphthalene input flux. The loss fraction rises with increasing naphthalene flux; at high input
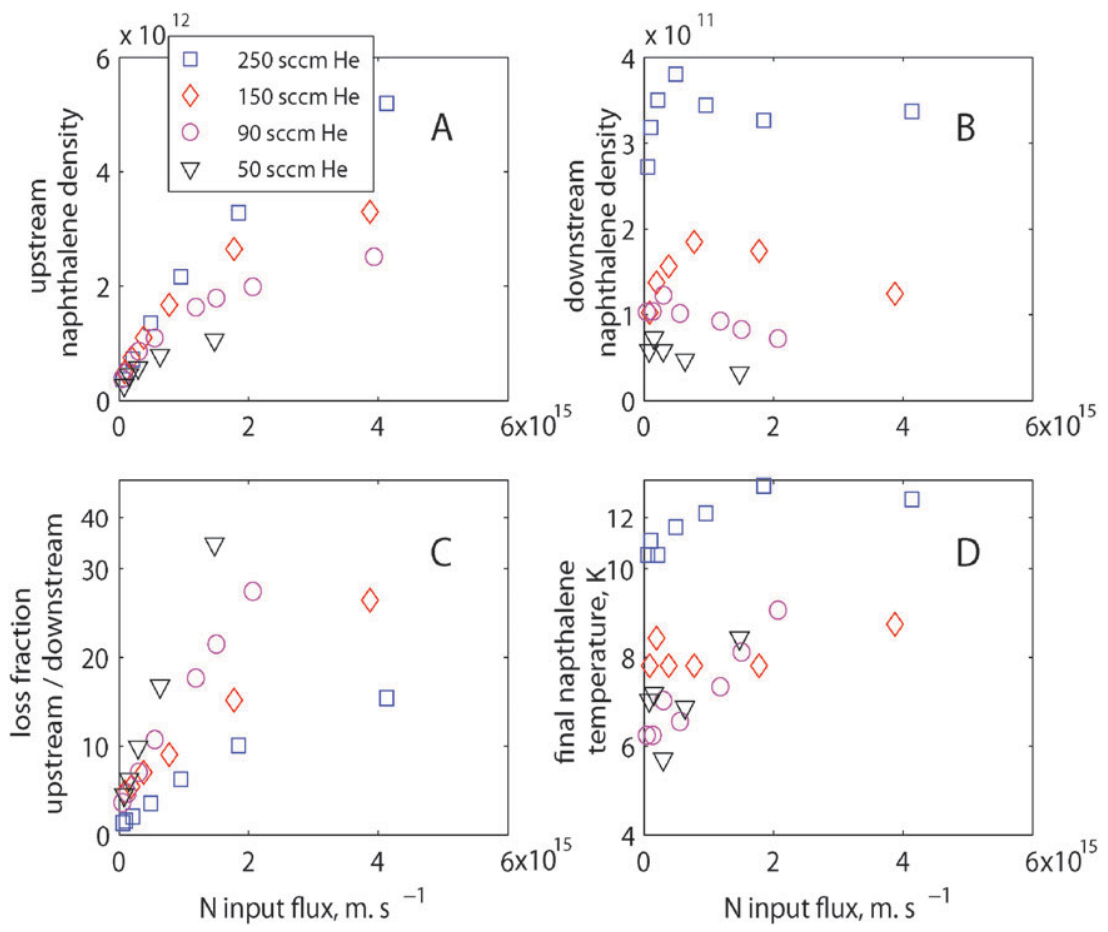

Fig. 3 Densities and temperatures of cold naphthalene under various conditions. Most of the cold naphthalene is lost as it is swept from the tube input ('upstream', subplot A) to the tube center ('downstream', subplot B) Loss in the tube (subplot C) clearly rises with increasing naphthalene input flux. The downstream temperature (subplot $\mathrm{D}$ ) is in reasonable thermodynamic equilibrium with the cold $(\approx 6.5 \mathrm{~K})$ cell for all but the highest helium flow rates. 
fluxes, increasing the naphthalene input actually results in a smaller downstream signal. As will be explained in detail below, this trend strongly suggests that the cold naphthalene is being consumed at low temperatures by reactions of the form

$$
\mathscr{N}+\mathscr{N} \Rightarrow \mathscr{N} \cdot \mathscr{N}
$$

or

$$
\mathscr{N}+\mathscr{N}_{n} \Rightarrow \mathscr{N}_{n+1}
$$

\section{$2.2 \mathscr{N} \cdot$ He and $\mathscr{N} \cdot \mathscr{N}$ dimerization}

Two key mechanisms for naphthalene loss are $\mathscr{N}$-He and $\mathscr{N}-\mathscr{N}$ dimerization. The binding energy of a helium atom to $\mathscr{N}\left(E_{\mathrm{He}-\mathscr{N}}\right)$ of $77 \mathrm{~cm}^{-1}$ is much higher than the $6 \mathrm{~K}\left(4.2 \mathrm{~cm}^{-1}\right)$ cell temperature. ${ }^{14}$ In complete thermal equilibrium, the fraction of naphthalene calculated to be naked (as opposed to dressed with one or more helium atoms) is only $10^{-4}$ (see Fig. 4). At higher ( $>15 \mathrm{~K}$ ) temperatures, even in equilibrium a very small fraction of the naphthalene molecules are expected to be "dressed"; complexes can therefore only form once the mixture is cold.

We undertook a careful spectroscopic search and observed no $\mathscr{N} \cdot$ He complexes in our system. The spectroscopy of these dimers $\left(\mathscr{N} \cdot \mathrm{He} \cdots \mathscr{N} \cdot \mathrm{He}_{n}\right.$, for $\left.n=1 \ldots 15\right)$, is known both experimentally $^{15}$ and studied theoretically. ${ }^{14}$ The center of the $8_{0}^{0}$ line in $\mathscr{N}$-He was measured in ref. 15 to be shifted by $1.6 \mathrm{~cm}^{-1}$ to the blue (from naked $\mathscr{N}$ ). We recorded spectra on the origin transition and observed no peak for $\mathscr{N} \cdot \mathrm{He}$, putting an upper bound of $10^{-2}$ on the $[\mathscr{N} \cdot \mathrm{He}] /[\mathscr{N}]$ fraction. We conclude that although we are in translational and rotational equilibrium, we are evidently collision rate limited in regards to $\mathscr{N} \cdot \mathrm{He}$ cluster formation. This allows us to determine a bound on the three body formation rate of these molecules of $R_{\mathscr{N}-\mathrm{He}-\mathrm{He}}<5 \times 10^{-35} \mathrm{~cm}^{6} \mathrm{~s}^{-1}$. We put an upper bound of $2 \%$

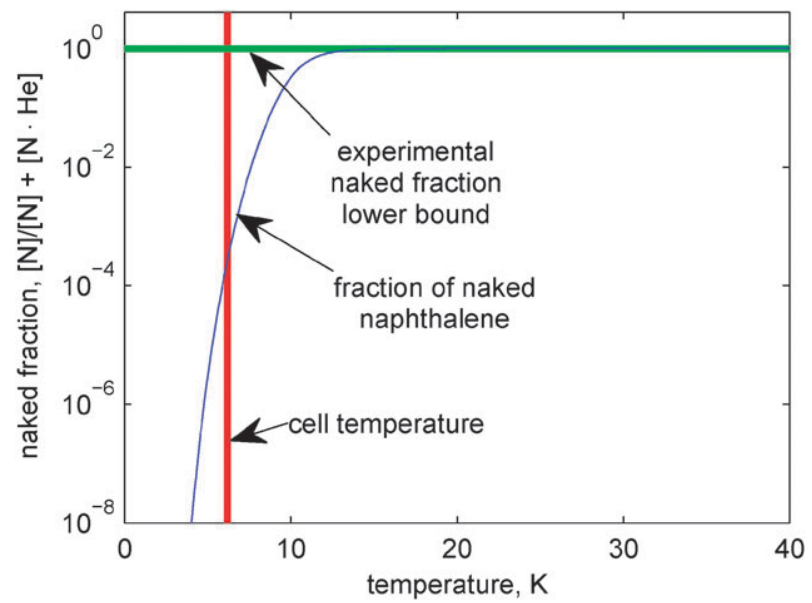

Fig. 4 The fraction of naked naphthalene expected in thermodynamic equilibrium as a function of temperature for a helium density of $n_{\mathrm{He}}=4 \times 10^{17} \mathrm{~cm}^{-3}$ (blue curve). The vertical line shows our experimental cell temperature of $T=6.2 \mathrm{~K}$, and the horizontal green line shows the experimental dimer fraction lower bound. No $\mathscr{N} \cdot \mathrm{He}$ dimers were observed. on the fraction of naphthalene molecules dressed with one or more helium atoms.

2.2.1 $\mathscr{N}$-He collisions. Conservation of energy and momentum precludes the reaction $\mathscr{N}+\mathrm{He} \Rightarrow \mathscr{N} \cdot \mathrm{He}$ from proceeding without the involvement of a third body.

Thus, the reaction must proceed either via a direct three body collision,

$$
\mathscr{N}+\mathrm{He}+\mathrm{He} \Rightarrow \mathrm{He} \mathscr{N}+\mathrm{He}
$$

or via the Lindemann mechanism, ${ }^{16}$ where the reaction proceeds in two steps, each a two body collision:

$$
\begin{gathered}
\mathrm{He}+\mathscr{N} \Rightarrow \mathrm{He} \mathscr{N}^{*} \\
\operatorname{He} \mathscr{N}^{*}+\mathrm{He} \Rightarrow \operatorname{He} \cdot \mathscr{N}+\mathrm{He}
\end{gathered}
$$

Where He $\mathscr{N}^{*}$ represents a quasibound complex. We consider the Lindemann mechanism first. If the complex $\mathrm{He} \mathscr{N}^{*}$ lives long enough to collide with a second helium atom before it dissociates, it is possible that one of the helium atoms will remain bound to the molecule, with the second helium atom carrying away the requisite energy and momentum to drive the reaction to completion. The effective three body recombination rate is therefore expected to be proportional to the lifetime of the He $\mathscr{N}^{*}$ complex. This lifetime is expected, in turn, to be a strong function of the number of internal degrees of freedom available for excitation in the system.

The lowest vibrationally excited level of naphthalene is $176 \mathrm{~cm}^{-1}$ above the origin, substantially above the maximum energy $E_{\mathrm{He}-\mathrm{n}}+O\left(k_{b} T\right) \approx 90 \mathrm{~cm}^{-1}$ available in the collision. From the point of view of naphthalene-helium collisions, the naphthalene molecule is therefore no different from a (slightly larger) atom, and the three body recombination rate should be comparable to that seen in atoms. Conservation of angular momentum during the collision is thought to prevent rotational degrees of freedom from playing a significant role in these processes $\nmid$ Our model suggests that it is the lowest vibrational mode $v_{\min }$ and the helium binding energy $E_{\mathrm{He}-\mathrm{X}}$, that are relevant when estimating Lindemann mechanism three body rates. For example, $\mathrm{C}_{60}$ is a large but exceptionally stiff molecule $\left(v_{\min }=232 \mathrm{~cm}^{-1}\right)$, while the amino acid tryptophan $\left(\mathrm{C}_{11} \mathrm{H}_{12} \mathrm{~N}_{2} \mathrm{O}_{2}\right)$ is not much larger than naphthalene, but contains low frequency torsional modes $\left(v_{\min }=46 \mathrm{~cm}^{-1}\right) .{ }^{17}$ Our model would therefore suggest that $\mathrm{C}_{60}$ could be cooled using this technique, but tryptophan could not. Other "stiff", and therefore coolable, candidates include the polar molecules benzonitrile $\left(v_{\min }=147 \mathrm{~cm}^{-1}\right)$ and azulene $\left(v_{\min }=167 \mathrm{~cm}^{-1}\right)$. The binding energy $E_{\mathrm{He}-\mathrm{X}}$ is also variable, but far less so, because the helium atom interacts only with nearby atoms. For example, helium binds to an infinite graphene sheet ${ }^{18}$ with an energy of $\sim 100 \mathrm{~cm}^{-1}$.

$\dagger$ An $\mathscr{N}$-He collision can certainly change the $\mathscr{N}$ molecule's angular momentum; in fact we rely on this process to rotationally cool the $\mathscr{N}$. However, a hypothetical bound $\mathrm{He} \mathscr{N}^{*}$ molecule produced in such a collision is constrained to have the same total angular momentum as the inputs; unlike vibration, the extra energy of the quasibound complex cannot be stored in the complex's rotation. For further discussion see ref. 12 . 
These conclusions are based on the Lindemann model of dimerization. We now turn to direct three body recombination processes that produce $\mathscr{N} \cdot \mathrm{He}_{n}$ clusters. In general, this mechanism in van der Waals complexes is poorly understood. In particular, for neutral particles these reactions have been observed only in the relatively uncontrolled conditions of a seeded supersonic jet. Simulations of these jets ${ }^{12}$ suggest that the $\mathscr{N}-\mathrm{He}-\mathrm{He}$ rate is on the order of $R_{\mathscr{N}-\mathrm{He}-\mathrm{He}}=$ $3 \times 10^{-35} \mathrm{~cm}^{6} \mathrm{~s}^{-1}$. We can compare this rate constant to other systems. For example, the strong chemical oxygen reaction $\left.\mathrm{O}+\mathrm{O}+\mathrm{O} \Rightarrow \mathrm{O}_{2}+\mathrm{O}\right)$ at $300 \mathrm{~K}$ has a much higher rate constant of $\sim 10^{-33} \mathrm{~cm}^{6} \mathrm{~s}^{-1}$.

The group of Kaneko et al. ${ }^{19}$ have observed the formation of rare gas ion-helium clusters (for example, $\mathrm{Ar}^{+} \cdot \mathrm{He}_{n}$ ), in cold helium gas $\left(n_{\mathrm{He}}=8 \times 10^{16} \mathrm{~cm}^{-3} T=4.4 \mathrm{~K}\right)$, via the Lindemann mechanism. They conclude that the net reaction $\mathrm{Ar}^{+} \mathrm{He}_{n}+\mathrm{He}+\mathrm{He} \Rightarrow \mathrm{Ar}^{+} \mathrm{He}_{n+1}+\mathrm{He}$ is suppressed in small clusters due to the short lifetime of the intermediate state $\mathrm{Ar}^{+} \mathrm{He}_{n+1}^{*}$. This is one of the few studies of three body recombination processes done at low temperature, but the $170 \mathrm{~cm}^{-1}$ binding energy of $\mathrm{Ar}^{+} \cdot \mathrm{He}$ and the lack of vibrational modes in this atomic system makes the applicability to the $\mathscr{N}$-He system questionable.

In our study, we observe no helium dimers even at our lowest temperatures $\left(T=6 \mathrm{~K} n_{\mathrm{He}}=4 \times 10^{17} \mathrm{~cm}^{-3}\right)$, putting an experimental upper bound on the rate at $R_{\mathscr{N} \text {-He-He}}$
$<5 \times 10^{-35} \mathrm{~cm}^{6} \mathrm{~s}^{-1}$, consistent with the results of experiments in supersonic jets.

2.2.2 $\mathscr{N}-\mathscr{N}$ collisions. Unlike $\mathscr{N} \cdot \mathrm{He}$ collisions, the binding energy of $E_{\mathcal{N}-\mathscr{N}}, 1008 \mathrm{~cm}^{-1},{ }^{20}$ is much larger than a typical naphthalene vibrational mode spacing of $\sim 300 \mathrm{~cm}^{-1}$. In addition, the $\mathscr{N}_{2}$ dimer has a rich internal vibrational spectrum, ${ }^{21}$ with a typical mode spacing for the complex of $\sim 20 \mathrm{~cm}^{-1}$. This suggests that the $\mathscr{N}+\mathscr{N}+\mathrm{He} \rightarrow \mathscr{N}_{2}+\mathrm{He}$ reaction is likely to proceed via effective two body processes:

$$
\begin{gathered}
\mathscr{N}+\mathscr{N} \Rightarrow \mathscr{N}_{2}^{*} \\
\mathscr{N}_{2}^{*}+\mathrm{He} \Rightarrow \mathscr{N}_{2}^{*}+\mathrm{He}
\end{gathered}
$$

We see strong evidence of this proposed two body loss from the cold sample. We further observe that this loss does not depend on the helium density and thus conclude that the lifetime of a $\mathscr{N}_{2}^{*}$ is substantially longer than the $\sim 10^{-8} \mathrm{~s}$ between helium collisions. Although direct observation of the expected $\mathscr{N}_{2}$ would be an ideal diagnostic of this process, the $\mathscr{N}_{2}$ dimer exhibits many geometrically distinct isomers and has an extremely broad spectral signal. In the future, detection of the $\mathscr{N}_{3}$ trimer, which has narrow lines, should be possible.

A simple model of loss initiatied by $\mathscr{N}-\mathscr{N}$ dimerization neatly explains all of our data on $\mathscr{N}$ loss. This model contains a single inelastic two body rate constant $k_{2}$, which characterizes all inelastic two body processes, and a single one body loss rate
Experiment
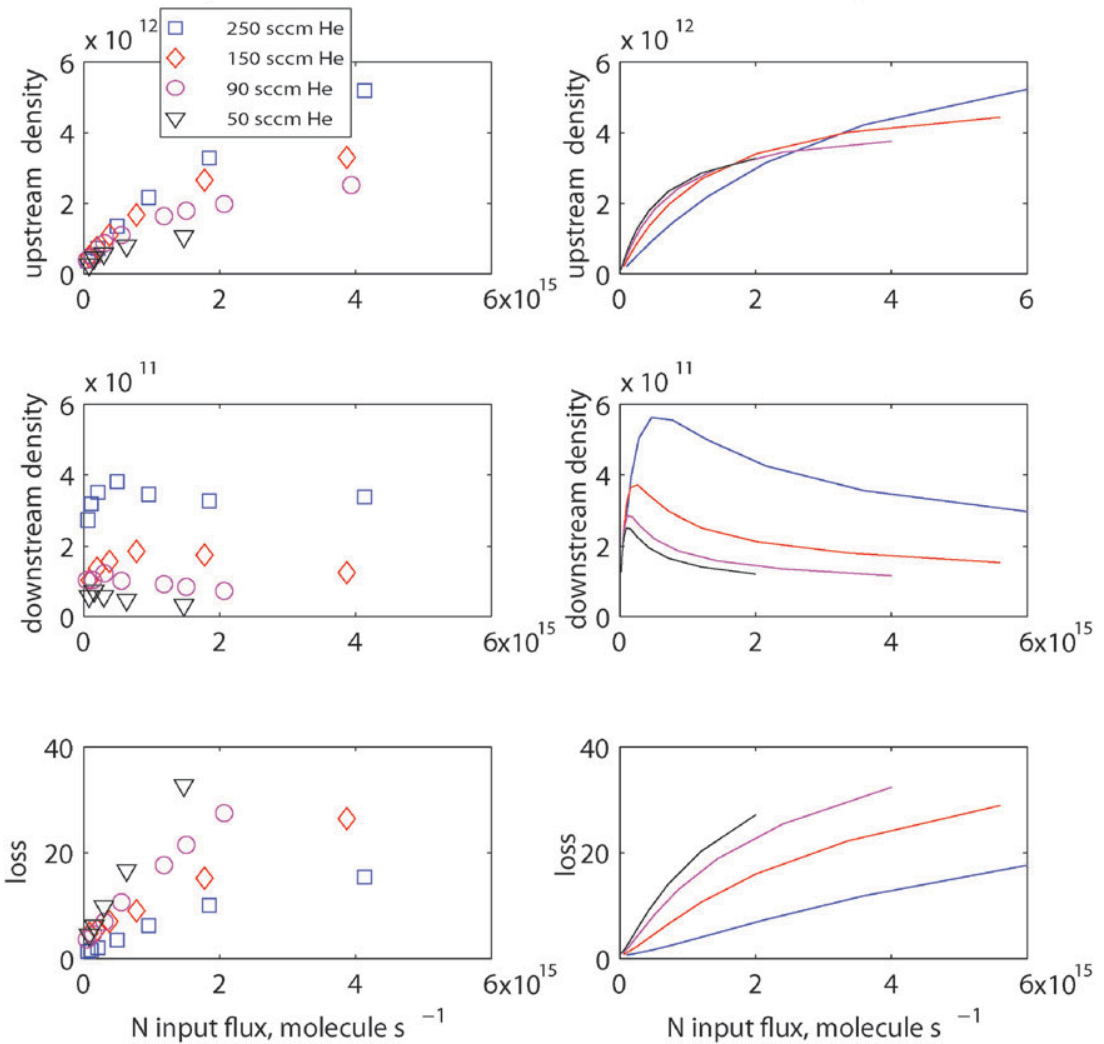

Fig. 5 The data from Fig. 3 (left hand plots) fit to a model that includes both two body loss and diffusion to the cell walls before and during the

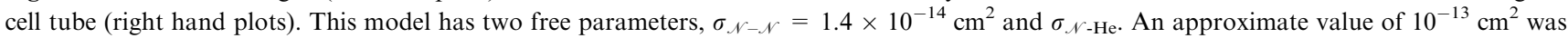
found for $\sigma_{\mathcal{N}-\mathrm{He}}$, although this value should be viewed with skepticism as it depends on the geometrical details of the buffer gas flow. 
$k_{1}$ which characterizes one body diffusion to the cell wall. If $[\mathscr{N}]$ is the density of naphthalene and $[\mathscr{M}]$ is the density of all dimers $\mathscr{N}_{n}$, this model can be written

$$
\begin{aligned}
& {[\dot{\mathcal{N}}]=-k_{2}[\mathscr{N}]^{2}-k_{2}[\mathscr{N}][\mathscr{M}]-k_{1}[\mathscr{N}]} \\
& {[\dot{\mathscr{M}}]=k_{2}[\mathscr{N}]^{2}-k_{2}[\mathscr{M}]^{2}-k_{1}[\mathscr{M}]}
\end{aligned}
$$

Although this model fits our data well, each reaction $\mathscr{N}_{x}+\mathscr{N}_{y} \Rightarrow \mathscr{N}_{x+y}$ must proceed at a unique rate, and the diffusion cross sections of $\mathscr{N}_{x}$ in helium will also vary. Nevertheless, the qualitative fit to the data, shown in Fig. 5, is good over a wide range of naphthalene and helium input fluxes. Our model suggests that under typical conditions, about $25 \%$ of the naphthalene input flux remains in gas phase at the point in the flow where the mixture has thermalized with the cold cell. The rest is lost, frozen on the cell and transition tube walls. This high efficiency of cooling is very likely not unique, as it depends only on the general cross section criteria $\sigma_{\mathscr{N} \text {-He }}>\sigma_{\mathrm{He}-\mathrm{He}}$ outlined earlier in this paper.

\section{Applications}

\subsection{Cold beam production}

Our method for large molecule injection could be combined with buffer-gas beam methods to produce cold, slow beams of large molecules. Earlier demonstrated buffer gas cooled beams ${ }^{1,22}$ include high-flux beams of $\mathrm{ND}_{3}$ and atomic potassium, produced by loading cold buffer gas cells using a similar methodology to this work. In those experiments, the mixture of buffer gas and molecules is sprayed out of the cell through a flat nozzle into a cryopumped vacuum so that the species of interest can be separated from the buffer gas via optical, electric, or magnetic fields. Replacing the pumping line described in this work with such a nozzle should produce a continuous, cold, high flux $\left(3 \times 10^{14}\right.$ molecule $\left.\mathrm{s}^{-1}\right)$ beam of naphthalene, or any similar sized molecule, in high vacuum. The beam would have a forward velocity of $\approx 150 \mathrm{~m} \mathrm{~s}^{-1}$, although related cold beam techniques could produce a substantially slower $\left(\approx 40 \mathrm{~m} \mathrm{~s}^{-1}\right)$ beam with a lower flux $\left(1 \times 10^{13}\right.$ molecule $\left.\mathrm{s}^{-1}\right){ }^{23}$ These techniques are extendable to larger polar molecules such as benzonitrile $\left(\mathrm{C}_{6} \mathrm{H}_{5} \mathrm{CN}\right.$, 4.2 Debye) or azulene, a polar isomer of naphthalene $\left(\mathrm{C}_{10} \mathrm{H}_{8}, 0.8\right.$ Debye $) .{ }^{24}$ Such a beam of larger polar molecules would make an attractive source for alternating gradient decelerators such as those demonstrated by Meijer et al. ${ }^{4}$

This cooling method is also promising as a tool for investigating cold chemistry. There has been much recent interest in cold gas-phase chemical reactions, including interest in chemistry in the interstellar medium, ${ }^{25}$ spin and orientation mediated chemical reactions, ${ }^{26}$ and field mediated reactions. The high densities, long interaction times (up to $50 \mathrm{~ms}$ in the current system), and generality of the cooling technique demonstrated here allow for a large variety of experiments, ranging from exploration of three body recombination to the possibility of field mediated reactions of aligned polar molecules.

\section{Conclusion}

Cold naphthalene has been produced in a $6 \mathrm{~K}$ vapor cell by rapidly cooling a warm mixture of helium and naphthalene. As the mixture cools, the phase space density of the naphthalene increases by more than five orders of magnitude, producing naphthalene at a $2 \times 10^{11} \mathrm{~cm}^{-3}$ density, $6.2 \mathrm{~K}$, and moving at a flow velocity of $1.5 \mathrm{~m} \mathrm{~s}^{-1}$. The samples remain in the cell for $\approx 50 \mathrm{msec}$, with typical loss dominated by the naphthalene-naphthalene dimerization reaction. Potential applications of the demonstrated technique to the study of cold chemistry, novel beam sources, and measurements of low temperature recombination are presented.

\section{References}

1 D. Patterson, J. Rasmussen and J. Doyle, New J. Phys., 2009, 11, 055018 (12pp).

2 L. D. Carr, D. DeMille, R. V. Krems and J. Ye, New Journal of Physics, 2009, 11, 055049 (87pp).

3 C. E. Heiner, H. L. Bethlem and G. Meijer, Phys. Chem. Chem. Phys., 2006, 8, 2666-2676.

4 J. Küpper, F. Filsinger and G. Meijer, Faraday Discuss., 2009, 142 155.

5 C. Sommer, L. van Buuren, M. Motsch, S. Pohle, J. Bayerl, P. Pinkse and G. Rempe, Faraday Discuss., 2009, 142, 203.

6 S. Deachapunya, P. J. Fagan, A. G. Major, E. Reiger, H. Ritsch, A. Stefanov, H. Ulbricht and M. Arndt, Eur. Phys. J. D, 2008, 46, 307.

7 D. H. Levy, Annu. Rev. Phys. Chem., 1980, 31, 197-225.

8 I. W. M. Smith and B. R. Rowe, Acc. Chem. Res., 2000, 33, 261.

9 L. Cai, J. Marango and B. Friedrich, Phys. Rev. Lett., 2001, 86, 775 .

10 F. Filsinger, J. Küpper, G. Meijer, L. Holmegaard, J. H. Nielsen, I. Nevo, J. L. Hansen and H. Stapelfeldt, J. Chem. Phys., 2009, 131, 064309.

11 F. Filsinger, J. Küpper, G. Meijer, J. Hansen, J. Maurer, J. H. Nielsen, L. Holmegaard and H. Stapelfeldt, Angew. Chem., Int. Ed., 2009, 48, 6900.

12 D. Patterson, PhD thesis, Harvard University, 2010.

13 W. Majewski and W. L. Meerts, J. Mol. Spectrosc., 1984, 104, 271-281.

14 E. Clementi and G. Corongiu, J. Phys. Chem. A, 2001, 105, 10379-10383.

15 U. Even, I. Al-Hroub and J. Jortner, J. Chem. Phys., 2001, 115, 2069-2074.

16 R. T. Pack, R. B. Walker and E. F. Hayes, Chem. Phys. Lett., 1997, 276, 255-262.

17 B. Yu, F. Zeng, Y. Yang, Q. Xing, A. Chechin, X. Xin, I. Zeylikovich and R. Alfano, Biophys. J., 2004, 86, 1649-1654.

18 M. C. Gordillo and J. Boronat, Phys. Rev. Lett., 2009, 102, 085303.

19 T. M. Kojima, N. Kobayashi and Y. Kaneko, Z. Phys. D, At., Mol. Clusters, 1992, 22, 645.

20 T. Fujiwara and E. C. Lim, J. Phys. Chem. A, 2003, 107, 4381-4386.

21 J. E. Wessel and J. A. Syage, J. Phys. Chem., 1990, 94, $737-747$.

22 L. D. van Buuren, C. Sommer, M. Motsch, S. Pohle, M. Schenk, J. Bayerl, P. W. H. Pinkse and G. Rempe, Phys. Rev. Lett., 2009, 102, 033001.

23 D. Patterson and J. Doyle, J. Chem. Phys., 2007, 126, 154307.

24 J. M. L. Martin, J. El-Yazal and J.-P. Franois, J. Phys. Chem., 1996, 100, 15358-15367.

25 E. Herbst, Аnпu. Rev. Phys. Chem., 1995, 46, $27-54$.

26 S. K. Kaesdorf, P. R. Brooks, R. F. Curl, J. H. Spence and S. J. Ulvick, Phys. Rev. A: At., Mol., Opt. Phys., 1986, 34, 4418. 